\title{
How Arch Support Insoles Help Persons with Flatfoot on Uphill and Downhill Walking
}

\author{
Yu-Ping Huang, ${ }^{1,2}$ Kwantae Kim, ${ }^{1}$ Chen-Yi Song, ${ }^{3}$ Yat-Hon Chen, ${ }^{4}$ and Hsien-Te Peng ${ }^{5}$ \\ ${ }^{1}$ Graduate Institute of Sport Coaching Science, Chinese Culture University, Taipei, Taiwan \\ ${ }^{2}$ School of Sports Science, Nantong University, Jiangsu, China \\ ${ }^{3}$ Department of Health Promotion and Gerontological Care, Taipei College of Maritime Technology, Taipei, Taiwan \\ ${ }^{4}$ Global Action Inc., Taipei, Taiwan \\ ${ }^{5}$ Department of Physical Education, Chinese Culture University, Taipei, Taiwan
}

Correspondence should be addressed to Hsien-Te Peng; sid125peng@yahoo.com.tw

Received 22 December 2016; Revised 4 March 2017; Accepted 15 March 2017; Published 9 April 2017

Academic Editor: Wenxin Niu

Copyright (C) 2017 Yu-Ping Huang et al. This is an open access article distributed under the Creative Commons Attribution License, which permits unrestricted use, distribution, and reproduction in any medium, provided the original work is properly cited.

\begin{abstract}
The main purpose of this study was to investigate the effect of arch support insoles on uphill and downhill walking of persons with flatfoot. Sixteen healthy college students with flatfoot were recruited in this study. Their heart rate, peak oxygen uptake $\left(\mathrm{VO}_{2}\right)$, and median frequency (MDF) of surface electromyogram were recorded and analyzed. Nonparametric Wilcoxon signed-rank test was used for statistical analysis. The main results were as follows: (a) peak $\mathrm{VO}_{2}$ significantly decreased with arch support insoles compared with flat insoles during uphill and downhill walking (arch support insole versus flat insole: uphill walking, $20.7 \pm 3.6$ versus $31.6 \pm 5.5$; downhill walking, $10.9 \pm 2.3$ versus $16.9 \pm 4.2$ ); (b) arch support insoles could reduce the fatigue of the rectus femoris muscle during downhill walking (MDF slope of arch support insole: $0.03 \pm 1.17$, flat insole: $-6.56 \pm 23.07$ ); (c) insole hardness would increase not only the physical sensory input but also the fatigue of lower-limb muscles particularly for the rectus femoris muscle (MDF slope of arch support insole: $-1.90 \pm 1.60$, flat insole: $-0.83 \pm 1.10$ ) in persons with flatfoot during uphill walking. The research results show that arch support insoles could effectively be applied to persons with flatfoot to aid them during uphill and downhill walking.
\end{abstract}

\section{Introduction}

Uphill and downhill walking exercise is considered a healthy recreational activity. Walking exercise is a popular activity worldwide. "Walking for Health" is the largest organization in England advocating for healthy walking. It consists of 70,000 walkers and encourages more than 63,000 people to engage in regular walking activity. This institution offers more than 3000 short-distance walks per week and provides a solution to the problem of sedentary behavior [1]. Hanson and Jones [2] showed that the benefits of walking exercise include reduction in systolic blood pressure, body mass index, and total cholesterol, among others. Previous studies have proved that walking exercise is beneficial for reducing blood pressure, reducing fasting glucose levels, and increasing $\mathrm{VO}_{2 \max }$ [3]. Moreover, Werner et al. [4] indicated that uphill walking with an inclination of $2 \%$ to $8 \%$ (equal to $1.2^{\circ}-4.6^{\circ}$ ) and constant velocity of each gait on an inclined treadmill can improve the symmetry of the human body. Nowadays, to relieve pressure at the workplace among urban workers, more and more people engage in walking exercise; however, persons with foot issues such as flatfoot are limited in their enjoyment of walking.

Persons with flatfoot have a foot arch support disability in which the midfoot collapses in the medial longitudinal side [5]. The midfoot functions as a shock absorber in the plantar portion, while it allows the foot arch to maintain appropriate elasticity in order to reduce the impact of the ground reaction force (GRF). Constant impact on the plantar aspect by the GRF increases the occurrence of injuries such as heel pain, pelvic malalignment, and plantar fasciitis [6]. Previous studies have identified that functional disorders in the foot could cause lower back, hip, knee, and ankle joint injuries $[7,8]$. Persons with flat feet might develop hallux valgus, plantar 
fasciitis, metatarsal pain, knee and back pain, and other problems without proper treatment [9]. Therefore, persons with flatfoot may not be able to sustain their body weight during prolonged uphill and downhill walking.

In prolonged walking, the arch support is important for mitigating plantar pressure and maintaining dynamic stability [10-13]. Previous studies reported that elderly persons with foot pain or leg symptoms who wore arch support insoles at a minimum duration of $4 \mathrm{~h} /$ day for 8 weeks showed improvement in standing balance and prevention of falls [10]. Saadah et al. [13] reported the effect of medial arch support in 16 hospital security guards during standing and walking before and after work and suggested that use of insole support to reduce the foot pressure and muscle work can strengthen the medial arch. Jafarnezhadgero et al. [14] indicated that walking with arch support insoles could reduce the impact of the vertical GRF by $6.9 \%$ while increasing the propulsion force by $7 \%$ and that the reduction in load rate through the arch support insoles could reduce the risk of lower-extremity injuries and damage caused by the GRF during walking.

Providing a solution, such as the use of arch support insoles, to facilitate participation in prolonged walking exercises and prevent lower-limb injuries is important in persons with flatfoot. Specifically, wearing arch support insoles might return flat feet to the condition of a normal arch. In order to examine the fatigue for people with flatfoot, the current study referred to previous researches which considered the heart rate [15], oxygen consumption $[15,16]$, and electromyography (EMG) $[15,16]$ as physical working capacity (PWC) of the fatigue thresholds. The purpose of this study was to investigate the effect of arch support insoles on the heart rate (HR), peak oxygen uptake $\left(\mathrm{VO}_{2}\right)$, and median frequency (MDF) of lower-limb muscles during uphill and downhill walking in persons with flatfoot. We hypothesized that the use of arch support insoles would lower the $\mathrm{HR}$, peak $\mathrm{VO}_{2}$, and MDF of lower-limb muscles.

\section{Materials and Methods}

2.1. Participants. The participants were 16 healthy college students (age: $18.3 \pm 0.7$ years, height: $167.5 \pm 6.4 \mathrm{~cm}$, weight: $65.1 \pm 14.4 \mathrm{~kg}$, body mass index: $23.2 \pm 4.7$, widest foot width: $8.5 \pm 0.7 \mathrm{~cm}$, and narrowest foot width: $6.1 \pm 1.1 \mathrm{~cm})$ with flatfoot (defined as an arch index (AI) of $72 \% \pm 10 \%$ ) $[17,18]$. All participants provided written informed consent. This study was approved by the Institutional Review Board of Antai Medical Care Corporation, Antai Tian-Sheng Memorial Hospital (TSMH, approval number 16-107-B1). The inclusion criteria required the absence of lower-limb injuries and of previous surgery in the lower limbs within the span of a year.

2.2. Experimental Protocol. Each participant's footprint was recorded with a footprint device (Footdisc Inc., Taipei, Taiwan) during 1 day of the experiment. Then, AI was calculated by using the narrowest foot width divided by the widest foot width from the footprint [17].
Another day was scheduled for the performance of the formal experiment. The participants were instructed to perform 15 min uphill and $15 \mathrm{~min}$ downhill walking randomly in standardized footwear (Maximizer16; Mizuno Taiwan Corporation, Taipei, Taiwan) with either a pair of arch support foot insoles (Footdisc) or a pair of flat insoles (Maximizer16, Mizuno Taiwan Corporation). The uphill and downhill walking was a simulated walking on a $\pm 9^{\circ}$ $[19,20]$ inclined treadmill (XG-1812X; New Noble Sport Equipment Co. Ltd., Ningbo, China) with a speed of $0.75 \mathrm{~m} / \mathrm{s}(2.7 \mathrm{~km} / \mathrm{h})[19,20]$. The hardness of the forefoot, midfoot, and heel of the insoles was measured with a hardness tester (Teclock GS-709N Type A; Teclock Co., Tokyo, Japan).

An HR monitor (H7; Polar Electro Inc., Kempele, Finland) was used to record the HR, and a portable spiroergometer (Metamax 3B; Cortex, Leipzig, Germany) was used to measure the peak $\mathrm{VO}_{2}$. Electromyogram (EMG) data were collected by using a Delsys system (Trigno wireless; Delsys Inc., Boston, MA, USA) with a $1000 \mathrm{~Hz}$ sampling rate.

The surface EMG (sEMG) sensors were adhered in parallel to the muscle belly of the subject's leg following the direction of muscle fibers. Before adhering the sensors on the muscles, the subjects stood tiptoe to mark the position of the gastrocnemius (GAS), did dorsiflexion to mark the position of the tibialis anterior (TA), did knee flexion to mark the position of the biceps femoris (BF), and did knee extension to mark the position of the rectus femoris (RF) with a black pen [21]. Then, the researchers shaved the participants' skin, removed hair impurities utilizing sandpaper, and cleaned with alcohol cotton sheet in order to gain better EMG signal. Additionally, we fixed the sensors on the leg using breathable tape to ensure the best conductivity and reduce the noise interference.

2.3. Data Processing. For dynamic contractions, MDF has been confirmed as a reliable indicator of muscle fatigue [22-24]. EMG spectrum will show lower signal expansion after muscle fatigue [23], and MDF will shift to the left of EMG spectral, that is, the MDF will decrease, which indicates the phenomenon of muscle fatigue $[25,26]$.

The raw data of EMG signals were converted into an MDF-time graph by using EMGWorks Analysis software (Delsys Inc., Boston, MA, USA) with a 0.125 s window length and $0.0124 \mathrm{~s}$ overlap. Then, the MDF-time graph was processed by using curve fit calculation (Figure 1). The slope of the curve was calculated to present the decrease/ increase of MDF during uphill and downhill walking. The formula of the MDF slope was $\left(Y_{2}-Y_{1}\right) /\left(X_{2}-X_{1}\right)$ (where $Y_{1}=$ first $\mathrm{MDF}$ value of the curve, $Y_{2}=$ smallest or last MDF value of the curve, $X_{1}=$ time of $Y_{1}$, and $X_{2}=$ time of $Y_{2}$ ).

2.4. Statistics. SPSS 18.0 (SPSS Science Inc., Chicago, IL, USA) for Windows was used for statistical calculations. Nonparametric Wilcoxon signed-rank test was used to compare the differences between arch support insole and flat insole in terms of $\mathrm{HR}$, peak $\mathrm{VO}_{2}$, and slope of $\mathrm{MDF}$ 


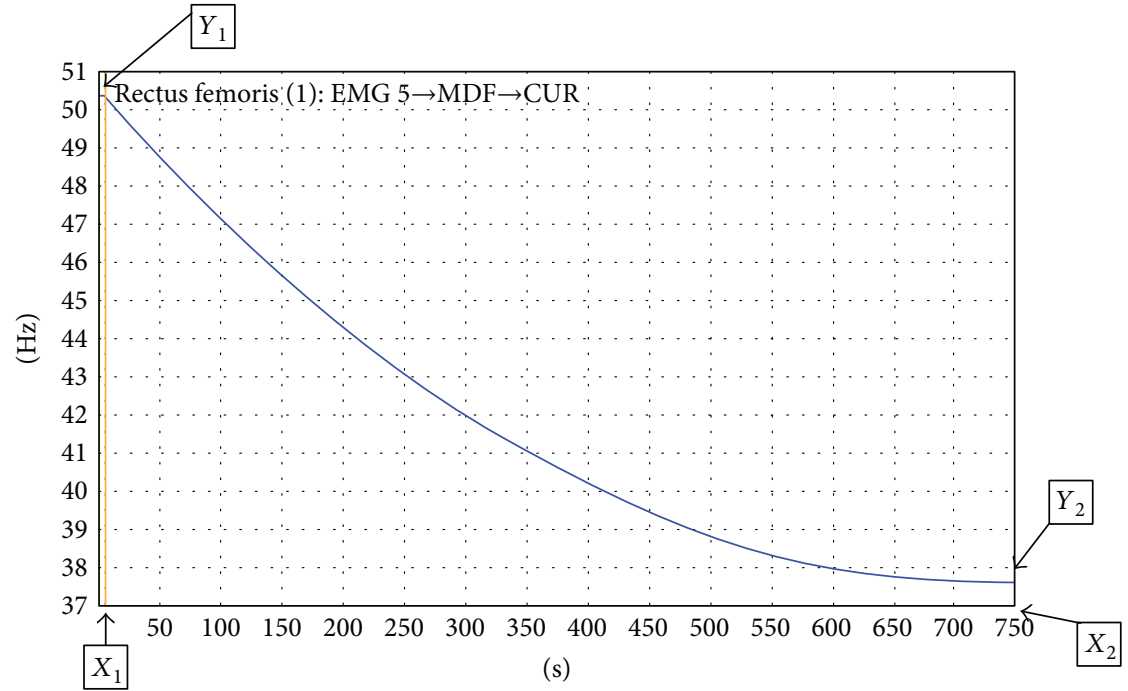

FIgURE 1: Graph after curve fitting.

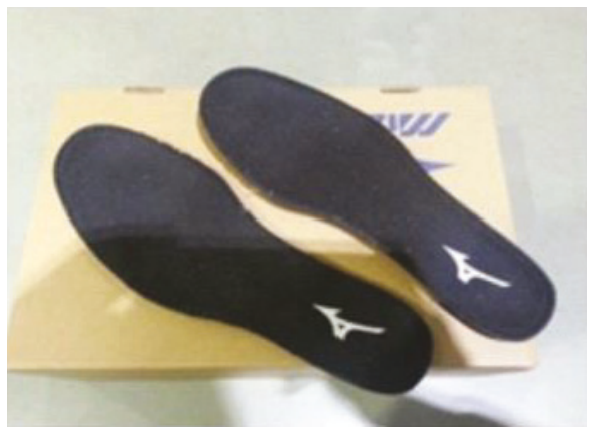

(a)

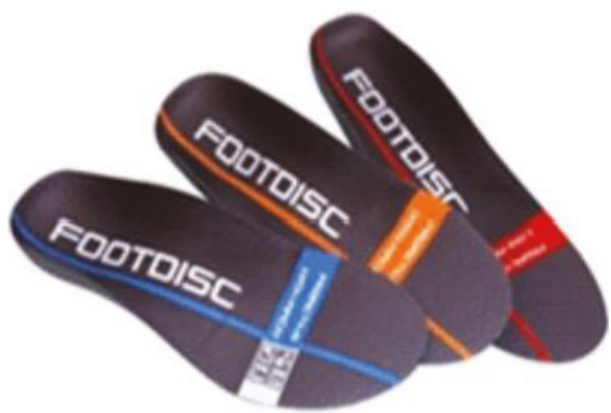

(b)

Figure 2: Flat insoles (a) and arch support insoles (b).

during uphill and downhill walking. The level of significance was set at $p<0.05$.

\section{Results}

3.1. Hardness of Insoles. The hardness of the forefoot, midsole, and heel areas was measured by using Teclock GS-709N Type A (Teclock Co.) for the two experimental conditions. The flat insole showed 35, 20, and 35 pointers, whereas the arch support insole showed 20, 60, and 20 pointers, respectively (Figure 2) (Table 1). In other words, the material of the arch support insole was harder than that of the flat insole in the midfoot region.

3.2. Peak Oxygen Uptake. Table 2 shows the outcomes of parameters during uphill and downhill walking. The peak $\mathrm{VO}_{2}$ showed significant differences between arch support insole and flat insole during both uphill and downhill walking (both $p<0.001$ ). The peak $\mathrm{VO}_{2}$ with arch support insole during both uphill and downhill walking was significantly smaller than that with flat insole based on positive ranks.

3.3. Median Frequency. The MDF slope of RF showed significant differences between arch support insole and flat
TABLE 1: Hardness of insoles.

\begin{tabular}{lcc}
\hline Areas & Arch support insole (pointer) & Flat insole (pointer) \\
\hline Forefoot & 20 & 35 \\
Midfoot & 60 & 20 \\
Heel & 20 & 35 \\
\hline
\end{tabular}

insole during uphill and downhill walking. During uphill walking, the MDF slope of RF with arch support insole $(-1.90 \pm 1.60 \mathrm{~Hz} / \mathrm{min})$ was significantly smaller than that with flat insole $(-0.83 \pm 1.10 \mathrm{~Hz} / \mathrm{min})$ (Table 2) based on positive ranks $(p=0.036)$. During downhill walking, the MDF slope of RF with flat insole $(-6.56 \pm 23.07 \mathrm{~Hz} / \mathrm{min})$ was significantly smaller than that with arch support insole $(0.03 \pm 1.17 \mathrm{~Hz} / \mathrm{min})$ based on negative ranks $(p=0.023)$. No difference was found in the HR and MDF slope of TA, $\mathrm{BF}$, and GAS.

\section{Discussion}

The primary findings of the present study indicated that the peak $\mathrm{VO}_{2}$ significantly decreased during both uphill and 
TABle 2: Parameter outcomes during uphill and downhill walking.

\begin{tabular}{lcccc}
\hline & \multicolumn{2}{c}{ Uphill walking } & \multicolumn{2}{c}{ Downhill walking } \\
& Arch support insole & Flat insole & Arch support insole & Flat insole \\
\hline Heart rate $(\mathrm{bpm})$ & $141.4 \pm 16.0$ & $140.9 \pm 14.4$ & $103.6 \pm 11.9$ & $105.1 \pm 12.6$ \\
${\text { Peak } \mathrm{VO}_{2}{ }^{*}\left(\mathrm{~mL} \mathrm{~min}{ }^{-1} \mathrm{~kg}^{-1}\right)}^{\text {MDF slope of muscles }}$ & $20.7 \pm 3.6^{\mathrm{a} *}$ & $31.6 \pm 5.5^{\mathrm{a} *}$ & $10.9 \pm 2.3^{\mathrm{a} *}$ & \\
$\quad$ & & & \\
Rectus femoris* $(\mathrm{Hz} / \mathrm{min})$ & $-1.90 \pm 1.60^{\mathrm{a} *}$ & $-0.83 \pm 1.10^{\mathrm{a} *}$ & $0.03 \pm 1.17^{\mathrm{b} *}$ & $-6.56 \pm 23.07^{\mathrm{b} *}$ \\
Tibialis anterior $(\mathrm{Hz} / \mathrm{min})$ & $-1.12 \pm 1.67$ & $-1.12 \pm 1.03$ & $-1.43 \pm 1.84$ & $-1.79 \pm 2.08$ \\
Biceps femoris $(\mathrm{Hz} / \mathrm{min})$ & $-1.23 \pm 1.73$ & $-1.21 \pm 0.99$ & $-0.79 \pm 1.57$ & $-1.54 \pm 0.93$ \\
Gastrocnemius $(\mathrm{Hz} / \mathrm{min})$ & $-1.38 \pm 1.63$ & $-1.03 \pm 1.25$ & $-1.34 \pm 2.25$ & $-2.01 \pm 1.72$ \\
\hline
\end{tabular}

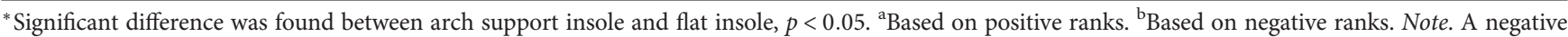
value of the MDF slope means a decrease of MDF.

downhill walking and that the decrease of the MDF of RF was significantly small only during downhill walking with arch support insole.

Wearing arch support insole could be beneficial for persons with flatfoot because their peak $\mathrm{VO}_{2}$, which represents the highest value of oxygen uptake in the span of $15 \mathrm{~min}$ uphill and 15 min downhill walking, decreased. Hreljac [27] indicated that an increase in exercise intensity, such as from walking to running, would lead to energy expenditure by the plantarflexor and dorsiflexor muscles. Haykowsky et al. [28] indicated that a high intensity of exercise would result in a significant increase of peak $\mathrm{VO}_{2}$ compared with moderate-intensity exercise. In other words, peak $\mathrm{VO}_{2}$ could be considered the intensity index of body loading. In the aspect of physiology, arch support insoles could reduce the loading of the human body. Therefore, persons with flatfoot who wear arch support insoles may be able to easily engage in the recreational exercise of uphill and downhill walking. We suggest that the arch support insole might effectively reduce the exercise loading due to the impact of uphill and downhill walking.

During downhill walking, RF showed more fatigue with flat insole than that with arch support insole. In previous researches, an effective EMG characteristic analysis for detection of muscle fatigue was based on the MDF, which would be smaller as the muscle fatigue increases [29, 30]. The MDF shift resulted from the change of the conduction velocity [31] and the change in intramuscular $\mathrm{pH}$ [32]. In the current study, when participants wore the arch support insole, a significantly lower decrease of MDF was observed only in RF muscle during downhill walking. However, a contrary outcome was found during uphill walking. It could be conjectured to be because of the different contraction types of RF during uphill and downhill walking. The contraction of the RF in uphill walking was considered to be concentric, whereas that in downhill walking was considered eccentric. In general, eccentric contraction was induced by a higher ground impact force compared with that in concentric contraction. Previous researchers indicated that activation of fast-twitch muscle fibers may be associated with a higher risk of injuries in eccentric contraction [33]. The arch support insole for persons with flatfoot could reduce RF fatigue, especially during downhill walking.
During uphill walking, RF showed more fatigue with arch support insole than that with flat insole. This outcome was in contrast to our hypothesis that the arch support insole should cause less muscle fatigue compared with the flat insole during uphill walking because the center of pressure is evenly redistributed on both feet owing to the arch support. Iglesias et al. [34] stated that increasing the insole hardness would increase the physical sensory input. Perry et al. [35] measured different midsole hardness conditions during walking along an $8 \mathrm{~m}$ walkway. They found that the range of the center of mass of the whole body increased (soft: $0.14 \mathrm{~m}$, hard: $0.16 \mathrm{~m}$ ) when the midsole hardness increased. A harder material of insole provides more strength for supporting the leg, which leads to more GRF and increases the range of the center of mass during walking. Yick et al. [36] also indicated that harder insoles would increase muscle activities. In other words, wearing arch support insoles not only could increase the leg support against GRF but also would cause more fatigue of the extremity muscles during uphill walking.

There are two limitations of this study. First, the $15 \mathrm{~min}$ uphill and downhill walking exercises were finished within 1 day. Gollhofer et al. [37] indicated that the movement in the conversion between concentric and eccentric contractions during exercise could reduce muscle fatigue; however, the conversion could cause damage to the muscles. The issue may happen to decrease the muscle fatigue during the slope walking in the current study. Second, a flexible flatfoot has an arch support on nonweight bearing but lost the arch support on weight bearing. A rigid flatfoot has loss of the longitudinal arch height [38]. The semirigid flatfoot means not much arch with and without pressure. They all have a common phenomenon which is no arch support on weight bearing. Therefore, in the current study, we recorded the footprint when participants were standing (weight bearing). Uphill and downhill walking are associated with the weight bearing (body weight). Therefore, we did not focus on those impacts of differences in the flexible flatfoot, rigid flatfoot, and semirigid flatfoot.

\section{Conclusion}

Wearing arch support insoles can be beneficial for uphill and downhill walking exercises in persons with flatfoot because 
the results of this study showed that oxygen uptake was effectively decreased during uphill and downhill walking, and there was less RF muscle fatigue during downhill walking.

\section{Conflicts of Interest}

The authors declare that there is no conflict of interest regarding the publication of this paper.

\section{Authors' Contributions}

The study was designed by Hsien-Te Peng and Yu-Ping Huang. Participant collection was supervised by Kwantae Kim and Yu-Ping Huang. Laboratory tests were performed by Kwantae Kim, Hsien-Te Peng, and Yu-Ping Huang. Data were collected and analyzed by Kwantae Kim and Hsien-Te Peng. Data interpretation and manuscript preparation were undertaken by Hsien-Te Peng, Yu-Ping Huang, Chen-Yi Song, and Yu-Ping Huang. All authors have read and approved the final version of the manuscript and agree with the order of presentation of the authors.

\section{References}

[1] J. South, "Health promotion by communities and in communities: current issues for research and practice," Scandinavian Journal of Public Health, vol. 42, Supplement 15, pp. 82-87, 2014.

[2] S. Hanson and A. Jones, "Is there evidence that walking groups have health benefits? A systematic review and metaanalysis," British Journal of Sports Medicine, vol. 49, no. 11, pp. 710-715, 2015.

[3] A. E. Tjønna, I. M. Leinan, A. T. Bartnes et al., "Low- and highvolume of intensive endurance training significantly improves maximal oxygen uptake after 10-weeks of training in healthy men," PloS One, vol. 8, no. 5, pp. 1-7, 2013.

[4] C. Werner, A. R. Lindquist, A. Bardeleben, and S. Hesse, "The influence of treadmill inclination on the gait of ambulatory hemiparetic subjects," Neurorehabilitation and Neural Repair, vol. 21, no. 1, pp. 76-80, 2007.

[5] M. Kido, K. Ikoma, K. Imai et al., "Load response of the medial longitudinal arch in patients with flatfoot deformity: in vivo 3D study," Clinical Biomechanics (Bristol, Avon), vol. 28, no. 5, pp. 568-573, 2013.

[6] J. Y. Jung, J. H. Kim, J. K. Choi, Y. Won, and J. J. Kim, "Association analysis of flat foot and high-arch foot using data mining," in Proceedings of the 12th International Conference on Applied Computer and Applied Computational Science, Kuala Lumpur, Malaysia, 2013.

[7] P. Harradine, L. Bevan, and N. Carter, "An overview of podiatric biomechanics theory and its relation to selected gait dysfunction," Physiotherapy, vol. 92, no. 2, pp. 122-127, 2006.

[8] A. R. Bird and C. B. Payne, "Foot function and low back pain," Foot (Edinburgh, Scotland), vol. 9, no. 4, pp. 175-180, 1999.

[9] C. R. Lee, M. K. Kim, and M. S. Cho, "The relationship between balance and foot pressure in fatigue of the plantar intrinsic foot muscles of adults with flexible flatfoot," Journal of Physical Therapy Science, vol. 24, no. 8, pp. 699-701, 2012.

[10] T. H. Chen, L. W. Chou, M. W. Tsai, M. J. Lo, and M. J. Kao, "Effectiveness of a heel cup with an arch support insole on the standing balance of the elderly," Clinical Interventions in Aging, vol. 9, pp. 351-356, 2014.

[11] F. Madehkhaksar and A. Egges, "Effect of dual task type on gait and dynamic stability during stair negotiation at different inclinations," Gait \& Posture, vol. 43, pp. 114-119, 2016.

[12] X. Qu, "Impacts of different types of insoles on postural stability in older adults," Applied Ergonomics, vol. 46, Part A, pp. 38-43, 2015.

[13] H. Saadah, D. Furqonita, and A. Tulaar, "The effect of medial arch support over the plantar pressure and triceps surae muscle strength after prolonged standing," Medical Journal of Indonesia, vol. 24, no. 3, pp. 146-149, 2015.

[14] A. Jafarnezhadgero, N. Farahpour, and M. Damavandi, "The acute effects of the application of arch support insole on ground reaction forces during walking," Journal of Research in Rehabilitation Sciences, vol. 11, no. 3, pp. 145-159, 2005.

[15] C. L. Camic, T. J. Housh, G. O. Johnson et al., "An EMG frequency-based test for estimating the neuromuscular fatigue threshold during cycle ergometry," European Journal of Applied Physiology, vol. 108, no. 2, pp. 337-345, 2010.

[16] K. C. Cochrane, T. J. Housh, H. C. Bergstrom et al., "Perceptual and physiological fatigue thresholds during cycle ergometry," Journal of Exercise Physiologyonline, vol. 17, no. 5, pp. 95-107, 2014, ISSN 1097-9751.

[17] F. Forriol and J. Pascual, "Footprint analysis between three and seventeen years of age," Foot \& Ankle International, vol. 11, no. 2, pp. 101-104, 1990.

[18] D. L. Riddiford-Harland, J. R. Steele, and L. H. Storlien, "Does obesity influence foot structure in prepubescent children?" International Journal of Obesity, vol. 24, no. 5, pp. 541-544, 2000.

[19] J. R. Franz and R. Kram, "The effects of grade and speed on leg muscle activations during walking," Gait \& Posture, vol. 35, no. 1, pp. 143-147, 2012.

[20] D. J. Haight, Z. F. Lerner, W. J. Board, and R. C. Browning, "A comparison of slow, uphill and fast, level walking on lower extremity biomechanics and tibiofemoral joint loading in obese and nonobese adults," Journal of Orthopaedic Research, vol. 32, no. 2, pp. 324-330, 2014.

[21] J. R. Cram, G. S. Kasman, and J. Holtz, Introduction to Surface Electromyography, Aspen Publishers, Gaithersburg, Maryland, 1998.

[22] M. R. Al-Mulla, F. Sepulveda, and M. Colley, "A review of noninvasive techniques to detect and predict localised muscle fatigue," Sensors, vol. 11, no. 4, pp. 3545-3594, 2011.

[23] D. R. Rogers and D. T. MacIsaac, "A comparison of EMGbased muscle fatigue assessments during dynamic contractions," Journal of Electromyography and Kinesiology, vol. 23, no. 5, pp. 1004-1011, 2013.

[24] M. Cifrek, S. Tonković, and V. Medved, "Measurement and analysis of surface myoelectric signals during fatigued cyclic dynamic contractions," Measurement, vol. 27, no. 2, pp. 85-92, 2000.

[25] D. Farina, M. Fosci, and R. Merletti, "Motor unit recruitment strategies investigated by surface EMG variables," Journal of Applied Physiology, vol. 92, no. 1, pp. 235-247, 2002.

[26] M. Cifrek, V. Medved, S. Tonković, and S. Ostojić, "Surface EMG based muscle fatigue evaluation in biomechanics," Clinical Biomechanics, vol. 24, no. 4, pp. 327-340, 2009. 
[27] A. Hreljac, "Preferred and energetically optimal gait transition speeds in human locomotion," Medicine and Science in Sports and Exercise, vol. 25, no. 10, pp. 1158-1162, 1993.

[28] M. J. Haykowsky, M. P. Timmons, C. Kruger, M. McNeely, D. A. Taylor, and A. M. Clark, "Meta-analysis of aerobic interval training on exercise capacity and systolic function in patients with heart failure and reduced ejection fractions," The American Journal of Cardiology, vol. 111, no. 10, pp. 1466-1469, 2013.

[29] S. Thongpanja, A. Phinyomark, P. Phukpattaranont, and C. Limsakul, "Mean and median frequency of EMG signal to determine muscle force based on time-dependent power spectrum," Elektron Elektrotech, vol. 19, no. 3, pp. 51-56, 2013.

[30] G. T. Allison and T. Fujiwara, "The relationship between EMG median frequency and low frequency band amplitude changes at different levels of muscle capacity," Clinical Biomechanics, vol. 17, no. 6, pp. 464-469, 2002.

[31] M. Solomonow, C. Baten, J. O. S. Smit et al., "Electromyogram power spectra frequencies associated with motor unit recruitment strategies," Journal of Applied Physiology, vol. 68, no. 3, pp. 1177-1185, 1990.

[32] B. Gerdle, S. Karlsson, A. G. Crenshaw, J. Elert, and J. Fridén, "The influences of muscle fibre proportions and areas upon EMG during maximal dynamic knee extensions," European Journal of Applied Physiology, vol. 81, no. 1, pp. 2-10, 2000.

[33] M. P. Mchugh, T. F. Tyler, S. C. Greenberg, and G. W. Gleim, "Differences in activation patterns between eccentric and concentric quadriceps contractions," Journal of Sports Sciences, vol. 20, no. 2, pp. 83-91, 2002.

[34] M. E. Losa Iglesias, R. Becerro de Bengoa Vallejo, and D. Palacios Peña, "Impact of soft and hard insole density on postural stability in older adults," Geriatric Nursing, vol. 33, no. 4, pp. 264-271, 2012.

[35] S. D. Perry, A. Radtke, and C. R. Goodwin, "Influence of footwear midsole material hardness on dynamic balance control during unexpected gait termination," Gait \& Posture, vol. 25, no. 1, pp. 94-98, 2007.

[36] K. L. Yick, L. T. Tse, W. T. Lo, S. P. Ng, and J. Yip, "Effects of indoor slippers on plantar pressure and lower limb EMG activity in older women," Applied Ergonomics, vol. 56, pp. 153-159, 2016.

[37] A. Gollhofer, P. V. Komi, M. Miyashita, and O. Aura, "Fatigue during stretch-shortening cycle exercises: changes in mechanical performance of human skeletal muscle," International Journal of Sports Medicine, vol. 8, no. 2, pp. 71-78, 1987.

[38] C. Napolitano, S. Walsh, L. Mahoney, and J. McCrea, "Risk factors that may adversely modify the natural history of the pediatric pronated foot," Clinics in Podiatric Medicine and Surgery, vol. 17, no. 3, pp. 397-417, 2000. 


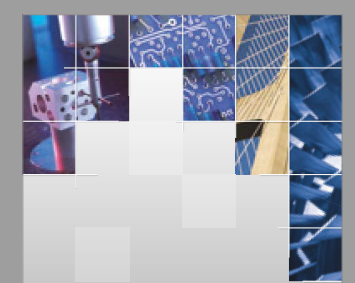

\section{Enfincering}
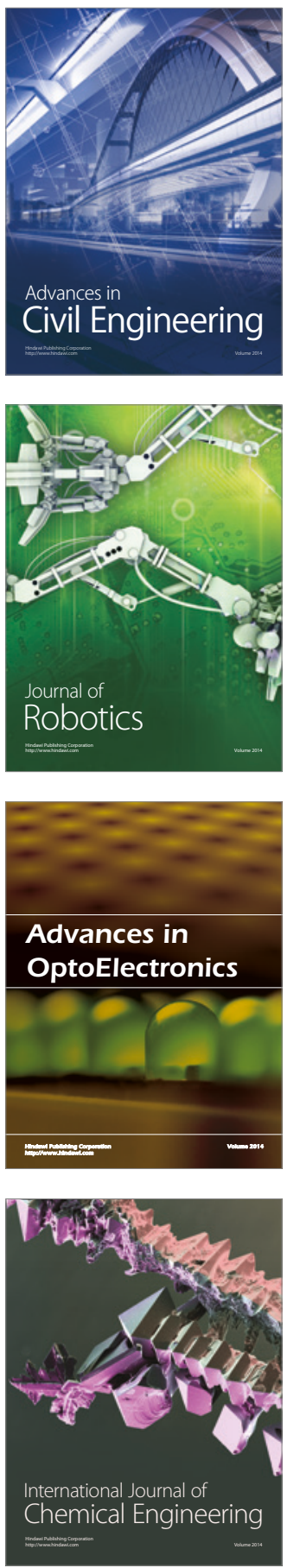

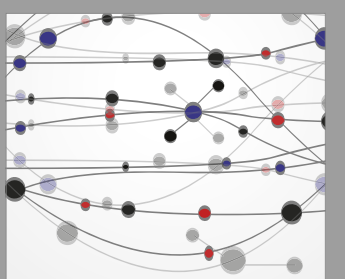

The Scientific World Journal

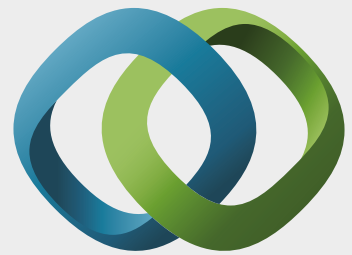

\section{Hindawi}

Submit your manuscripts at

https://www.hindawi.com
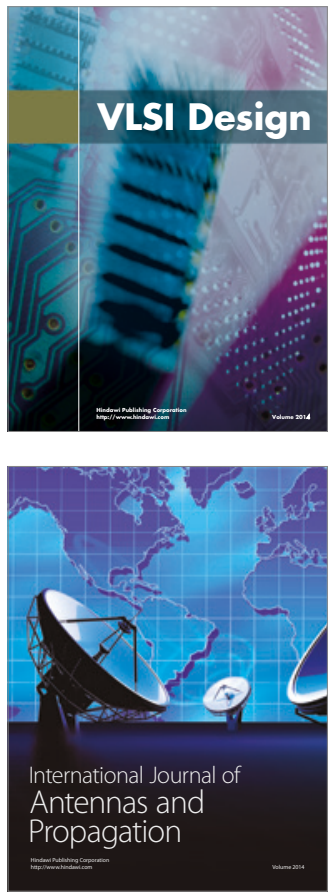

\section{Rotating}

Machinery
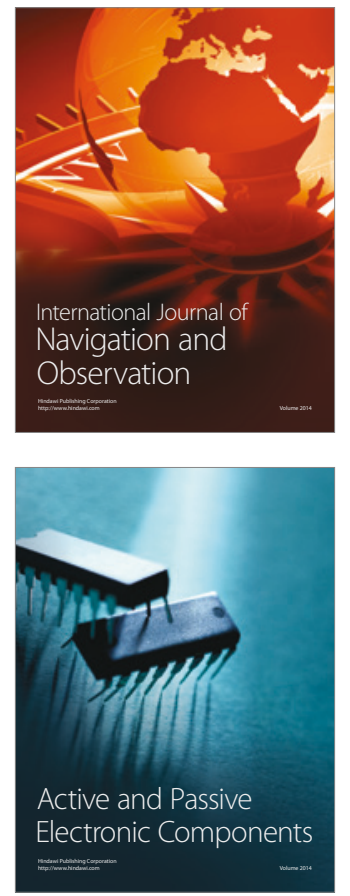
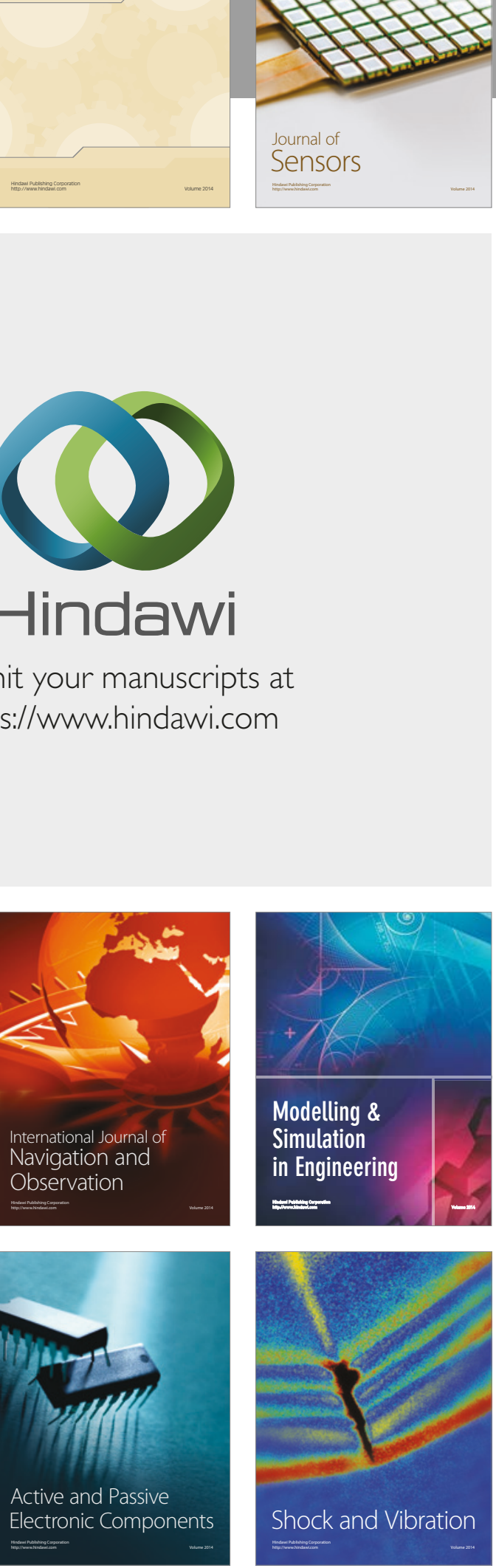
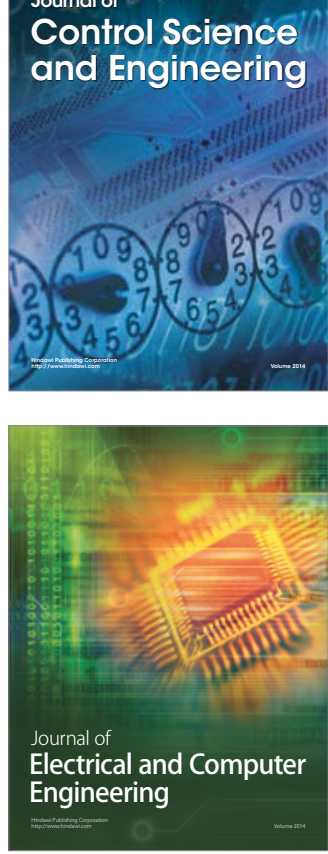

Distributed

Journal of

Control Science

and Engineering
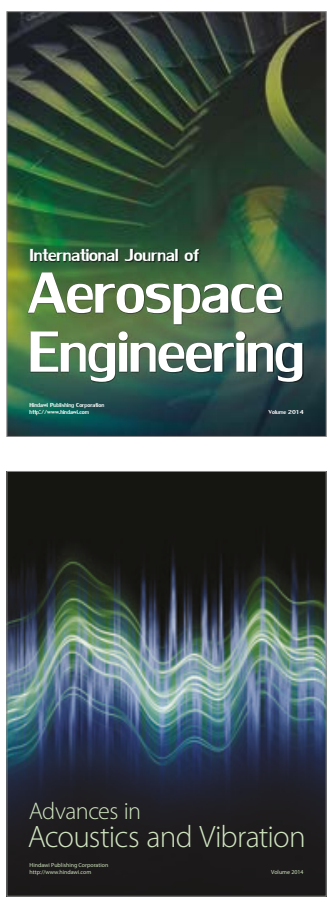

Sensor Networks 